\title{
Editorial
}

\section{Thin Film Transistor}

\author{
Ray-Hua Horng \\ Institute of Electronics, National Chiao Tung University, Hsinchu 30010, Taiwan; rhh@nctu.edu.tw
}

Received: 25 July 2019; Accepted: 9 August 2019; Published: 9 August 2019

\begin{abstract}
The special issue is "Thin Film Transistor". There are eight contributed papers. They focus on organic thin film transistors, fluorinated oligothiophenes transistors, surface treated or hydrogen effect on oxide-semiconductor-based thin film transistors, and their corresponding application in flat panel displays and optical detecting. The present special issue on "Thin Film Transistor" can be considered as a status report reviewing the progress that has been made recently on thin film transistor technology. These papers can provide the readers with more research information and corresponding application potential about Thin Film Transistors.
\end{abstract}

Keywords: thin film transistor; OTFT; surface treated; hydrogen effect; flat panel displays; optical detecting

Thin film transistor (TFT) is a type of field effect transistors whose active layer, the current-carrying layer, is a thin film made by depositing an active semiconductor layer, as well as the dielectric layer over a non-conducting substrate. In general, TFTs are made at low material cost and processed at low temperature. Moreover, fabrication time of TFT is shorter than that of traditional MOSFETs. TFTs have been demonstrated in a wide variety of applications, such as active-matrix liquid-crystal displays (LCD), active-matrix organic light-emitting displays, photodetecting devices, and biosensors. A display comprises a matrix of the smallest addressable element called pixels. An image on the display is formed by thousands (millions) of these pixels arranged in rows and columns. The TFTs act as switches which allow the pixels to turn on and off very easily. Another promising area of application is the biosensing system where TFT arrays perform electrical excitation and sensing for the study of biological cells. In TFT biosensor, a sensitive layer converts biochemical signals into electrically measurable physical parameters.

Organic thin film transistors (OTFTs) employ organic material as the semiconductor layer, and are a promising alternative to traditional mainstream inorganic TFT. The OTFTs are well suited with flexible plastic substrates at low temperatures and equivalent with inorganic TFT in basic operation and design. The effect of device geometry on performance parameter of OTFT is examined by Arnal et al. [1]. In their work, the same material and fabrication procedure is applied to two different transistor geometries-corbino and interdigitated. Corbino geometry has less parasitic capacitance as they provide less overlapping area in comparison to the interdigitated shape. The organic inverter circuit produced by using two OTFT shows maximum gain and lowest hysteresis with a Corbino structure as the driver and an interdigitated structure as the load. Krammer et al. [2] proposed a two-step fitting method to analyze transistor model with the experimental current-voltage characteristic of OTFT. First, the output and transfer characteristic of OTFT is fitted to transistor model and the parameters of the transistor are extracted. In the second step, the dependency of extracted parameters and the channel length is determined. This two-step method gives better results by comparing calculated and measured output characteristics but fails to verify channel-length-dependence of the transistor parameters. Chang, J.-F., et al. [3] examined the effect of microstructural and molecular growth of $\alpha, \omega$-diperfluorohexylquaterthiophene (DFH-4T) film on charge transport. The field-effect mobility increases significantly with the deployment of DFH-4T an n-type material in organic field-effect 
transistors. With the increase of DFT-4T thickness from 8 to $80 \mathrm{~nm}$, mobility increases from 0.01 to $1 \mathrm{~cm}^{2} . \mathrm{V}^{-1} \cdot \mathrm{s}^{-1}$, and the threshold voltage first decreases then increases.

The surface treatment is an important method used to remove contaminants and improve the performance of the semiconductor device. Horng, R. -H., et al. [4] demonstrated a chemical solution treatment on the $\mathrm{n}-\mathrm{GaN}$ surface before $\mathrm{Al}_{2} \mathrm{O}_{3}$ was grown on metal-oxide-semiconductor (MOS); capacitor consists of an $\mathrm{Al}_{2} \mathrm{O}_{3} / \mathrm{n}-\mathrm{GaN} / \mathrm{AlN}$ buffer/Si substrate. The chemical treatments containing oxygen plasma, $\mathrm{BCl}_{3}$ plasma, dilute acidic/ alkali solvents, and hydrofluoric acid were used to improve the surface quality and reduce interface state trap density. Several works have reported the surface treatment of the dielectric layer to improve the TFT performance. Most of the charge carriers are confined to the semiconductor film adjacent to the semiconductor/dielectric interface. The charge carrier transport is affected by characteristics of the dielectric surface. The surface treatment of dielectric significantly improves the majority carrier mobility and reduces the interface trap.

Wide bandgap oxide semiconductor has been widely investigated due to their high mobility and low leakage currents. Among oxide semiconductor devices, amorphous indium-gallium-zinc-oxide $(\alpha$-IGZO) thin film transistors (TFTs) are strong candidates for next-generation flat-panel displays and active-matrix organic light-emitting diode (AMOLED) display applications. However, various defects exist between metal and oxygen, which affects the performance of the device. In addition, the electrical characteristics of $\alpha$-IGZO TFT are affected by the presence of hydrogen impurity. Hydrogen generates free carrier in the oxide semiconductor and also acts as defect passivation. Noh, H. -Y., et al. [5] investigated the effect of hydrogen injection on the $\alpha$-IGZO system at different oxygen environments. Depending on the oxygen environments, hydrogen species reduces or increases defect states. The physical mechanism of optical devices based on transparent conductive oxide (TCO) such as In-Ga-Zn-O, In-Zn-O was reviewed by Jang, J., et al. [6]. By introducing oxygen defects into TCO, an optical memory action called persistent photoconductivity (PPC) is eluded. In advanced neuromorphic system, a TCO based device with PPC phenomenon could be used as optical synaptic TFT. Overall, TCO could play an important role in transparent display and low-cost optoelectronic application.

The TFT-LCDs play a prominent role in high-resolution flat panel displays. The drain-source contact and pixel electrode layer of TFT controls the light switching function and the frame rate of the LCD. An automatic optical inspection (AOI) system performs a quality check of these layers by employing a light source. The performance of three light sources metal-halide lamps, quartz-halogen lamps, and LEDs for scanning pixel electrode of TFT was investigated by Tzu, F. -M., et al. [7]. From the result, it was found that LED is a better choice in terms of cost and performance as compared to the quartz-halogen lamp and metal-halide lamp. Due to better spatial resolution and spectrum compatibility, LED should be adopted in AOI by considering energy efficiency and performance. The defect in LCD can be detected by the human eye as it can perceive green light of spectrum $555 \mathrm{~nm}$. Tzu, F. -M., et al. [8] demonstrated machine vision associated with transmission chromaticity spectrometer to detect non-uniformity of the green emission layer for TFT-LCD. The just noticeable difference was utilized as the detection criterion, according to that of the International Commission on Illumination. The presented method is an alternative to manual optical inspection as it quantifies the defects more accurately.

The present Special Issue on "Thin Film Transistor" can be considered as a status report reviewing the progress that has been made recently on thin film transistor technology.

\section{References}

1. Arnal, A.; Martínez-Domingo, C.; Ogier, S.; Terés, L.; Ramon, E. Monotype Organic Dual Threshold Voltage Using Different OTFT Geometries. Crystals 2019, 9, 333. [CrossRef]

2. Krammer, M.; Borchert, J.W.; Petritz, A.; Karner-Petritz, E.; Schider, G.; Stadlober, B.; Klauk, H.; Zojer, K. Critical Evaluation of Organic Thin-Film Transistor Models. Crystals 2019, 9, 85. [CrossRef] 
3. Chang, J.-F.; Shie, H.-S.; Yang, Y.-W.; Wang, C.-H. Study on Correlation between Structural and Electronic Properties of Fluorinated Oligothiophenes Transistors by Controlling Film Thickness. Crystals 2019, 9, 144. [CrossRef]

4. Horng, R.-H.; Tseng, M.-C.; Wuu, D.-S. Surface Treatments on the Characteristics of Metal-Oxide Semiconductor Capacitors. Crystals 2018, 9, 1. [CrossRef]

5. Noh, H.Y.; Kim, J.; Kim, J.-S.; Lee, M.-J.; Lee, H.-J. Role of Hydrogen in Active Layer of Oxide-Semiconductor-Based Thin Film Transistors. Crystals 2019, 9, 75. [CrossRef]

6. Jang, J.; Kang, Y.; Cha, D.; Bae, J.; Lee, S. Thin-Film Optical Devices Based on Transparent Conducting Oxides: Physical Mechanisms and Applications. Crystals 2019, 9, 192. [CrossRef]

7. Tzu, F.-M.; Chou, J.-H. Effectiveness of Light Source on Detecting Thin Film Transistor. Crystals 2018, 8, 394. [CrossRef]

8. Tzu, F.-M.; Chou, J.-H. Optical Detection of Green Emission for Non-Uniformity Film in Flat Panel Displays. Crystals 2018, 8, 421. [CrossRef]

(C) 2019 by the author. Licensee MDPI, Basel, Switzerland. This article is an open access article distributed under the terms and conditions of the Creative Commons Attribution (CC BY) license (http://creativecommons.org/licenses/by/4.0/). 